\title{
Corrigendum
}

\section{On uniquely implementing cooperation in the prisoners' dilemma}

\author{
Bhaskar Chakravorti ${ }^{1}$, John Conley ${ }^{2}$ and Bart Taub ${ }^{2}$ \\ 1 The Monitor Company, Cambridge, MA 02141, USA \\ ${ }^{2}$ Department of Economics, University of Illinois, Champaign, IL 61820, USA
}

Received: August 23, 1996

In the main text of our recent paper [1], we demonstrated how restricting players to stationary strategies resulted in unique Pareto-dominant equilibria. In the appendix we asserted that broadening the definition of stationarity to mean any set of strategies representable by a Markov transition process and enforced by a grim trigger duplicated the result, implying that infinitely complicated strategies were necessary to generate the folk theorem outcome. (Recall that the folk theorem states that any individually rational payoffs are an equilibrium if players are sufficiently patient.) While the assertions of the main text remain valid, in fact the folk theorem emerges with very simple, finite-state Markov strategies. In particular, any 2-state (and therefore finite) oscillating strategy can generate the folk theorem; moreover, the folk theorem equilibrium set emerges at the same discount factor that yields the unique Pareto dominant equilibrium under the stationarity restriction - in our parlance, the critical $\delta$.

The result follows easily from noting that the equilibrium equation from the appendix,

$$
\left(\mathcal{T}^{2}-\left(\lambda^{*} D\right)^{-1} I\right) x=0
$$

has two solutions for $n=2$. One is the stationary solution we discussed in which $\mathcal{T}$ is the identity matrix, which remains valid. A second, which we overlooked, is to set

$$
\mathcal{T}=\left(\begin{array}{ll}
0 & 1 \\
1 & 0
\end{array}\right)
$$

which represents oscillation between two states. The square of $\mathcal{T}$ is the identity, and it therefore solves the above equation. At the critical value of 
$\delta, \lambda D=1$, and therefore

$$
\lambda D \mathcal{T} x=X
$$

so that

$$
X=\left(\begin{array}{l}
X_{1} \\
X_{2}
\end{array}\right)=\left(\begin{array}{l}
x_{2} \\
x_{1}
\end{array}\right)
$$

representing oscillating strategies. Any individually rational combination of $x_{1}$ and $x_{2}$ works, thus yielding the folk theorem.

The result replicates the discontinuities that appear in the equilibrium set as a set function of the discount factor that appear in the papers we cited: [2], [3], and [4]. Thus, we can now state that these discontinuities are the result of allowing only slightly complicated strategies. (In the folk theorem of the main text, Theorem 1, which covers the $n=1$ case, a lower value of $\delta$ is needed; moreover, the equilibrium set is continuous with respect to $\delta$.) If there are any costs of changing strategies, we conjecture that such equilibria will be dominated, leaving the stationary equilibria we explored in the main text as the unique equilibria.

\section{References}

1. Chakravorti, B., Conley, J., Taub, B.: On uniquely implementing cooperation in the repeated prisoners' dilemma. Economic Theory 8, 347-366 (1996)

2. Sorin, S.: On repeated games with complete information. Mathematics of Operations Research 11, 147--161 (1986)

3. Stahl, D.: The graph of prisoners' dilemma supergame payoffs as a function of the discount factor. Games and Economic Behavior 3 (1991)

4. Van Damme, E.: Stability and perfection of Nash equilibria. Springer, Berlin Heidelberg New York (1991) 